\title{
High-pressure structural phase transitions in $\mathrm{CuWO}_{4}$
}

\author{
J. Ruiz-Fuertes, ${ }^{1, *}$ D. Errandonea,,${ }^{1,2}$ R. Lacomba-Perales, ${ }^{1}$ A. Segura, ${ }^{1}$ J. González,,${ }^{3,4}$ F. Rodríguez, ${ }^{3}$ F. J. Manjón, ${ }^{5}$ \\ S. Ray, ${ }^{5}$ P. Rodríguez-Hernández, ${ }^{6}$ A. Muñoz,${ }^{6}$ Zh. Zhu, ${ }^{7}$ and C. Y. Tu ${ }^{7}$ \\ ${ }_{1}^{1}$ Departamento de Física Aplicada-ICMUV, Universitat de València, Edificio de Investigación, c/Dr. Moliner 50, 46100 Burjassot, Spain \\ ${ }^{2}$ Fundación General Universitat de València, Edificio de Investigación, c/Dr. Moliner 50, 46100 Burjassot, Spain \\ ${ }^{3}$ DCITIMAC, Universidad de Cantabria, Avda. de Los Castros s/n, 39005 Santander, Spain \\ ${ }^{4}$ Centro de Estudios de Semiconductores, Universidad de los Andes, Mérida 5201, Venezuela \\ ${ }^{5}$ Instituto de Diseño para la Fabricación y Producción Automatizada, Universidad Politécnica de Valencia, \\ Camino de Vera s/n, 46022 Valencia, Spain \\ ${ }^{6}$ Departamento de Física Fundamental II, Instituto de Materiales y Nanotecnología, \\ Universidad de La Laguna, La Laguna, 38205 Tenerife, Spain \\ ${ }^{7}$ Fujian Institute of Research on the Structure of Matter, Chinese Academy of Sciences, Fujian, Fuzhou, People's Republic of China
}

(Received 3 May 2010; revised manuscript received 11 June 2010; published 25 June 2010)

\begin{abstract}
We study the effects of pressure on the structural, vibrational, and magnetic behavior of cuproscheelite. We performed powder x-ray diffraction and Raman spectroscopy experiments up to $27 \mathrm{GPa}$ as well as ab initio total-energy and lattice-dynamics calculations. Experiments provide evidence that a structural phase transition takes place at $10 \mathrm{GPa}$ from the low-pressure triclinic phase $(P \overline{1})$ to a monoclinic wolframite-type structure $(P 2 / c)$. Calculations confirmed this finding and indicate that the phase transformation involves a change in the magnetic order. In addition, the equation of state for the triclinic phase is determined: $V_{0}=132.8(2) \AA^{3}, B_{0}$ $=139(6) \mathrm{GPa}$, and $B_{0}^{\prime}=4$. Furthermore, experiments under different stress conditions show that nonhydrostatic stresses induce a second phase transition at $17 \mathrm{GPa}$ and reduce the compressibility of $\mathrm{CuWO}_{4}, B_{0}$ $=171(6) \mathrm{GPa}$. The pressure dependence of all Raman modes of the triclinic and high-pressure phases is also reported and discussed.
\end{abstract}

DOI: 10.1103/PhysRevB.81.224115

PACS number(s): 62.50.-p, 61.50.Ks, 61.05.cp, 64.30.Jk

\section{INTRODUCTION}

Since the discovery of high-temperature superconductivity and its relation with the coordination of $\mathrm{Cu}$ with $\mathrm{O}$ atoms and the linking of these polyhedra to networks, the study of the crystal structure of $\mathrm{Cu}$ compounds has experienced an increased interest. ${ }^{1}$ Although quaternary oxides are in the center of interest, the effects of pressure in the $\mathrm{Cu}$ environment in systems such as $\mathrm{CuWO}_{4}$ could be helpful in the search of routes for superconductivity. Several high-pressure (HP) structural studies have been performed in compounds related with $\mathrm{CuWO}_{4}{ }^{2-4}$ However, due to its low crystal symmetry, the crystallographic study under pressure has shown to be more complicated.

Copper tungstate $\left(\mathrm{CuWO}_{4}\right.$, the mineral cuproscheelite) is a member of the wolframite series of structurally related materials. It crystallizes in a triclinic structure $(P \overline{1})$ (Ref. 5) with both cations octahedrally coordinated by $\mathrm{O}$ atoms. The $\mathrm{CuO}_{6}$ octahedra present a Jahn-Teller (JT) distortion that gives rise to an approximately elongated octahedron. This causes a distortion of the lattice that produces the twofold axes and mirror planes disappearance, lowering the crystal symmetry from $P 2 / c$ (wolframite) to $P \overline{1}$. Since the refinement of the $\mathrm{CuWO}_{4}$ structure, ${ }^{5}$ several structural and vibrational studies have been carried out in $\left(\mathrm{Zn}_{x} \mathrm{Cu}_{1-x}\right) \mathrm{WO}_{4}$ solid solutions. ${ }^{6-9}$ It is known that a ferroelastic phase transition from cuproscheelite to wolframite takes place at $x=0.75$. More recently, optical and Raman measurements ${ }^{10,11}$ provided evidence on the existence in $\mathrm{CuWO}_{4}$ of a phase transition near $10 \mathrm{GPa}$. However, there is no structural information on the high-pressure phase and only part of the Raman modes have been explored under compression up to $16 \mathrm{GPa}$. The aim of this work is to study the compressibility and structural phase transitions, as well as further explore the vibrational and magnetic properties of cuproscheelite. In order to achieve this goal, we performed angle-dispersive x-ray diffraction (ADXRD) and Raman spectroscopy studies under different stress conditions. The experimental studies are combined with $a b$ initio total-energy and lattice-dynamics calculations.

\section{EXPERIMENTAL DETAILS}

We performed two independent x-ray diffraction experiments. In one experiment (exp. 1), silicone oil (SO) was used as pressure-transmitting medium. This experiment was performed at beamline I15 in the Diamond Light Source with a monochromatic x-ray beam $(\lambda=0.61506 \AA)$, which was focused down to $30 \mu \mathrm{m} \times 30 \mu \mathrm{m}$ using K-B mirrors. A membrane-type diamond-anvil cell (DAC) with $400-\mu \mathrm{m}$-diameter culet diamonds and a $180-\mu \mathrm{m}$-drilled Inconel gasket was used. In the other experiment (exp. 2), argon (Ar) was the pressure medium. This experiment was carried out with a symmetric DAC with 480 - $\mu$ m-diameter culet diamonds and a 150- $\mu \mathrm{m}$-drilled rhenium gasket, at 16IDB beamline of the HPCAT at the Advanced Photon Source (APS). In this case, a monochromatic $\mathrm{x}$-ray beam $(\lambda$ $=0.36783 \AA$ ) was focused down to $10 \mu \mathrm{m} \times 10 \mu \mathrm{m}$. In both experiments, the diffraction patterns were recorded on a MAR345 image plate located at 450 and $350 \mathrm{~mm}$ from the sample, respectively, and integrated using FIT2D. To perform the experiments, we used micron-size powders with purity 
higher than $99.5 \%$ (Mateck). The pressure was measured by means of the ruby fluorescence technique. In the second experiment, pressure was confirmed with the equation of state (EOS) of Ar. ${ }^{12}$ Prior to loading the DACs, x-ray diffraction and Raman measurements confirmed that only the lowpressure triclinic phase is present in the samples. The unitcell parameters were $a=4.709(7) \AA, b=5.845(9) \AA, c$ $=4.884(7) \AA, \alpha=88.3(2)^{\circ}, \beta=92.5(2)^{\circ}$, and $\gamma=97.2(2)^{\circ}$, in agreement with Ref. 5. The indexation of the Bragg reflections was done with UNITCELL and DICVOL. GSAS was used to carry out Le Bail refinements ${ }^{13}$ of the low- and high-pressure structures.

Two Raman studies were performed in 10 - $\mu$ m-thick platelets cleaved from a single crystal. The single-crystal preparation was described in Ref. 10. For these measurements, we used a membrane-type DAC with 500- $\mu \mathrm{m}$-diameter culet diamonds and a 200- $\mu \mathrm{m}$-drilled Inconel gasket. A 16:3:1 methanol-ethanol-water (MEW) mixture and SO were used as pressure-transmitting media. Pressure was determined using the ruby fluorescence scale. In one experiment, the Raman spectra was measured up to 17 $\mathrm{GPa}$ with the $647.1 \mathrm{~nm}$ line of a Coherent krypton laser (model Innova 300) using a Ramanor U1000 double monochromator equipped with a liquid-nitrogen refrigerated Symphony charge coupled device (CCD) detector. The Raman spectra under pressure were obtained in second order with an attached confocal microscope. In the second experiment, measurements up to 21.1 GPa were performed in a backscattering geometry using a LabRam HR UV microRaman spectrometer with a 1200 grooves/mm grating and $100 \mu \mathrm{m}$ slit, in combination with a thermoelectric-cooled multichannel CCD detector. A He-Ne $632.81 \mathrm{~nm}$ laser line with a power below $10 \mathrm{~mW}$ was used for Raman excitation to avoid thermal effects since the usage of higher laser power showed the appearance of burned areas in the sample. The silicon Raman mode $\left(520 \mathrm{~cm}^{-1}\right)$ was used as a reference for the calibration of the Raman spectra. In both measurements, the spectral resolution was below $2 \mathrm{~cm}^{-1}$.

\section{CAlCulation TECHNIQUE}

$A b$ initio total-energy and lattice-dynamics calculations were done within the framework of the density-functional theory (DFT) and the pseudopotential method using the Vienna $a b$ initio simulation package (VASP), ${ }^{14}$ a first-principles plane-wave code which can describe the exchange and correlation energy in the local-density approximation (LDA) and in the generalized-gradient approximation (GGA). A detailed account can be found in Refs. 15-18. All our calculations are performed at $T=0 \mathrm{~K}$. The exchange and correlation energy was initially taken in the GGA according to PerdewBurke-Ernzerhof prescription. ${ }^{19}$ The projector-augmented wave (PAW) scheme se $^{20}$ was adopted and the semicore $5 p$ electrons of $\mathrm{W}$ were dealt with explicitly in the calculations. The set of plane waves used extended up to a kinetic-energy cutoff of $520 \mathrm{eV}$. This large cutoff was required to deal with the $\mathrm{O}$ atoms within the PAW scheme to ensure highly converged results. The Monkhorst-Pack ${ }^{21}$ grid used for Brillouin-zone integrations ensured highly converged results for the analyzed structures (to about $1 \mathrm{meV} / \mathrm{f} . \mathrm{u}$.). We performed spin-density calculations and we found that the antiferromagnetic (AF) configuration was the stable one for the low-pressure triclinic phase $(P \overline{1})$ whereas the ferromagnetic (FM) configuration was the most stable for the monoclinic wolframite-type structure $(P 2 / c)$. At each selected volume, we optimized the atomic geometry including ionic coordinates, the structures were fully relaxed to their equilibrium configuration through the calculation of the HellmanFeynman forces on atoms and the stress tensor, see Ref. 22. In the relaxed configurations, the forces are less than $0.006 \mathrm{eV} / \AA$ and the deviation of the stress tensor from a diagonal hydrostatic form is less than $0.1 \mathrm{GPa}$. The highly converged results on forces are required for the calculation of the dynamical matrix using the direct force-constant approach (or supercell method). ${ }^{23}$ The construction of the dynamical matrix at the $\Gamma$ point is quite simple and involves separate calculations of the forces in which a fixed displacement from the equilibrium configuration of the atoms within the primitive unit cell is considered. Symmetry aids by reducing the number of such independent distortions and reducing the amount of computational effort in the study of the analyzed structures considered in our work. Diagonalization of the dynamical matrix provides both the frequencies of the normal modes and their polarization vectors, it allows us to identify the irreducible representation and the character of the phonon modes at the zone center. In the paper, we will comment on the pressure dependence of the Raman-active modes of the different structures. For completion, we report the frequencies and pressure coefficients of the IR-active modes for the triclinic and monoclinic phases in Appendix.

\section{RESULTS AND DISCUSSION}

\section{A. X-ray diffraction}

In Figs. 1 and 2, we show selected x-ray diffraction patterns of ADXRD experiments 1 and 2 performed with SO and Ar, respectively. In both figures, it can be observed that up to $7.8 \mathrm{GPa}$ all the Bragg reflections can be indexed according to the $\mathrm{CuWO}_{4}$ triclinic structure (phase I). Beyond 9 $\mathrm{GPa}$, extra peaks appear (depicted by arrows in the two figures) pointing out the onset of a phase transition, to a phase with monoclinic symmetry that we will denote as phase II, in good agreement with previous Raman and optical-absorption experiments. ${ }^{11}$ As we will explain later, the triclinic structure coexists with the monoclinic phase II during more than 6 $\mathrm{GPa}$ after the transition onset. Note that in experiment 2, one peak of the fcc structure of $\mathrm{Ar}$ is identified at about $2 \theta$ $=7.6^{\circ} .{ }^{12}$ This peak is depicted with a star and is easy to identify since its pressure shift is different from that of the $\mathrm{CuWO}_{4}$ peaks. In experiment 2, we do not find any evidence of additional structural changes or chemical decomposition of $\mathrm{CuWO}_{4}$ up to $20.3 \mathrm{GPa}$. In contrast, in experiment 1 , beyond $16 \mathrm{GPa}$ there is an extinction of the peaks of the triclinic phase and additional peaks are detected. In particular, a new peak located around $2 \theta=7.5^{\circ}$ and another one around $2 \theta=11.5^{\circ}$ can be clearly seen in the figure at 20.3 $\mathrm{GPa}$. Both facts indicate the onset of a second transition to a 


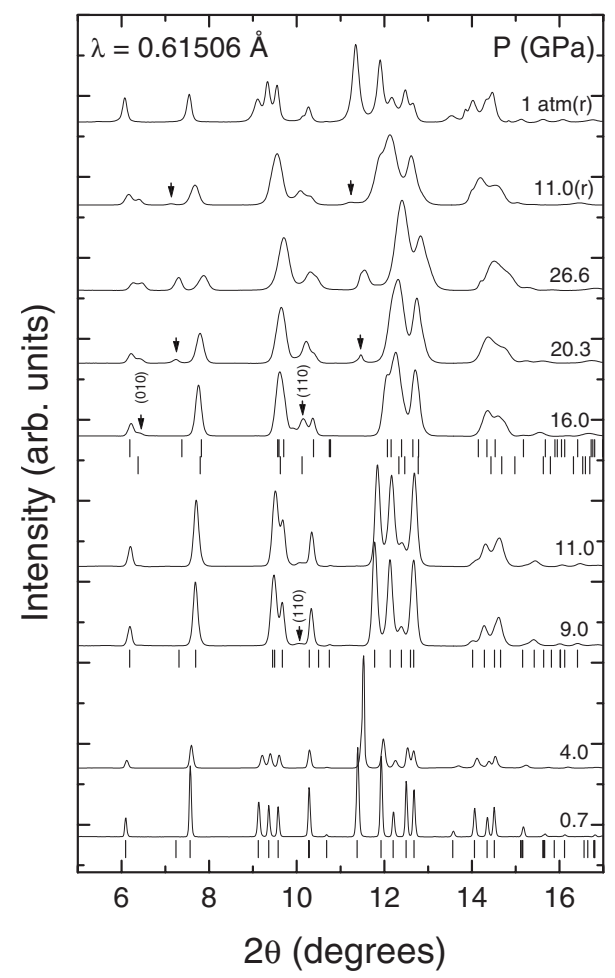

FIG. 1. Selected x-ray diffraction patterns of the experiment performed using silicone oil as pressure-transmitting medium. The ticks indicate the identified Bragg reflections of different structures. Distinctive peaks of the wolframite phase are labeled and the emerging peaks related to phase III are indicated with an arrow (at $20.3 \mathrm{GPa}$ ).

phase that we will name phase III. The detected structural changes are reversible in both experiments as can be seen in Figs. 1 and 2. However, even though the triclinic phase is fully recovered after pressure release, phase III is present on decompression up to $8 \mathrm{GPa}$ when $\mathrm{SO}$ is the pressuretransmitting medium. We will see later that Raman measurements fully support this observation and allow us to confirm that only one phase transition takes place upon decompression from phase III to I.

From the analysis of all the diffraction patterns, we have obtained the pressure behavior of the lattice parameters of the low-pressure phase of $\mathrm{CuWO}_{4}$ (phase I). A Le Bail refinement has been used to fit the X-ray diffraction profiles (see Fig. 3 and Table I) and the normalized unit-cell parameters are reported as a function of pressure up to $10 \mathrm{GPa}$ in Fig. 4. We observe that the $c$ axis is less compressible than the other two axes. This anisotropic compression is higher in the experiment performed under Ar. Similar anisotropic behaviors were found also in the structurally related $\mathrm{CdWO}_{4}$, $\mathrm{MgWO}_{4}, \mathrm{MnWO}_{4}$, and $\mathrm{ZnWO}_{4} \cdot{ }^{4,24}$ This fact is related to the different linking of octahedral units along different crystallographic directions, being the $\mathrm{CuO}_{6}$ octahedra much more compressible than the $\mathrm{WO}_{6}$ octahedra. Figure 5 shows the pressure dependence of the unit-cell volume which was analyzed using a second-order Birch-Murnaghan EOS $\left(B_{0}^{\prime}=4\right) .{ }^{25}$ The unit-cell volume $\left(V_{0}\right)$ and the bulk modulus $\left(B_{0}\right)$ at zero pressure obtained for the triclinic phase are $V_{0}$ $=132.8(2) \AA^{3}$ for both experiments, and $B_{0}=171(2)$ and

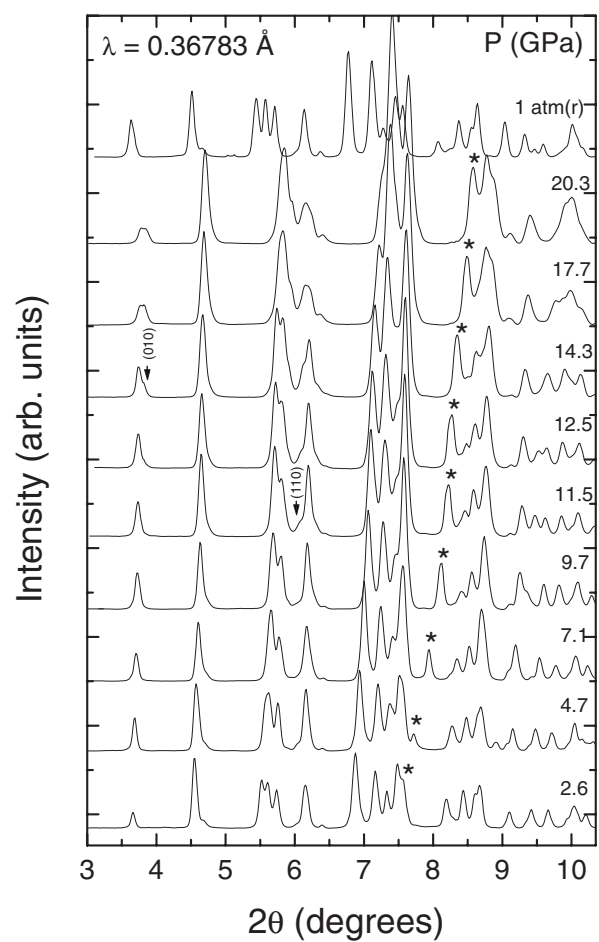

FIG. 2. Selected x-ray diffraction patterns of the experiment performed using argon as pressure-transmitting medium. The asterisks indicate the Ar Bragg peak. The (110) and (010) Bragg reflections of the HP wolframite phase are also indicated.

134(6) GPa for experiments 1 and 2, respectively. The fitted EOSs are shown as lines in Fig. 5. The obtained bulk moduli agree with the value calculated using the empirical model proposed in Ref. 26, which gives an estimated value of 158 $\mathrm{GPa}$. The bulk modulus of $\mathrm{CuWO}_{4}$ between 139 and 171 $\mathrm{GPa}$ is also comparable with those obtained in wolframitestructure tungstates. ${ }^{4}$

Our experiments show that $\mathrm{CuWO}_{4}$ is $18 \%$ more compressible using Ar than using SO. This medium-dependent behavior found in $\mathrm{CuWO}_{4}$ can be explained if we have into account that $\mathrm{Ar}$ is a better hydrostatic medium than $\mathrm{SO}{ }^{27,28}$

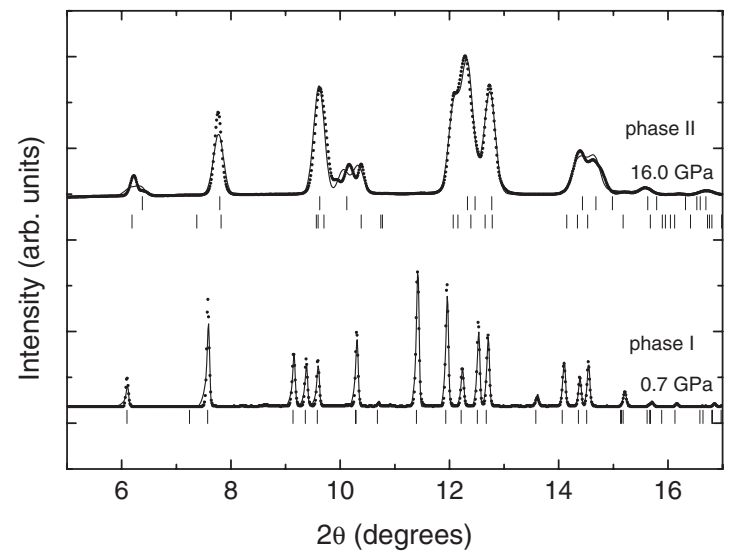

FIG. 3. Le Bail fit of diffraction patterns of phases I (0.7 GPa) and II (16 GPa). Dots: experiments. Lines: model. Ticks indicate the position of calculated Bragg reflections. The lattice parameters and angles at these pressures are given in Table I. 
TABLE I. Structural parameters of the triclinic and monoclinic structures of $\mathrm{CuWO}_{4}$ and different pressure.

\begin{tabular}{lcccccccc}
\hline \hline Structure & $\begin{array}{c}\text { Pressure } \\
(\mathrm{GPa})\end{array}$ & $\begin{array}{c}a \\
(\AA)\end{array}$ & $\begin{array}{c}b \\
(\AA)\end{array}$ & $\begin{array}{c}c \\
(\AA)\end{array}$ & $\begin{array}{c}\alpha \\
(\mathrm{deg})\end{array}$ & $\begin{array}{c}\beta \\
(\mathrm{deg})\end{array}$ & $\begin{array}{c}\gamma \\
(\mathrm{deg})\end{array}$ & $\begin{array}{c}\text { Volume } \\
\left(\AA^{3}\right)\end{array}$ \\
\hline$P \overline{1}$ & 0.7 & 4.695 & 5.827 & 4.876 & 91.627 & 92.385 & 83.004 & 132.23 \\
& 16 & 4.523 & 5.716 & 4.783 & 90.831 & 89.910 & 85.566 & 123.26 \\
$P 2 / c$ & 16 & 4.524 & 5.529 & 4.896 & 90 & 90.861 & 90 & 122.44 \\
\hline \hline
\end{tabular}

It is well known that nonhydrostatic effects can influence the structural properties of a material if its mechanical strength is smaller than the one of ruby (such as $\left.\mathrm{CuWO}_{4}\right) .{ }^{29}$ Indeed, there are many examples in the literature of ternary oxides (e.g., $\mathrm{ZrSiO}_{4}$ and $\mathrm{CaWO}_{4}$ ) (Refs. 26 and 30) where bulk modulus differences of up to $20 \%$ are found depending on hydrostaticity despite being highly uncompressible materials. Usually, larger bulk moduli are systematically obtained with the less hydrostatic media ${ }^{31}$ a fact that agrees with our findings for $\mathrm{CuWO}_{4}$. The nonhydrostatic effects could be also responsible for the second phase transition detected in the experiment done under $\mathrm{SO}$, a fact that we confirmed by Raman spectroscopy. It has been observed in tungstates such as $\mathrm{BaWO}_{4}$ and $\mathrm{PbWO}_{4}$ that the structural sequence is very sensitive to any degree of nonhydrostaticity. ${ }^{32}$ Therefore, apparently nonhydrostatic conditions induced by the use of MEW and $\mathrm{SO}$ as pressure-transmitting media, ${ }^{27}$ make $\mathrm{CuWO}_{4}$ less compressible and favor the occurrence of a second transition. The influence of nonhydrostatic stresses can be also seen in the inset of Fig. 5. There, it is shown that the Bragg peaks considerably broaden under compression beyond $16 \mathrm{GPa}$ when SO is the pressure medium. In addition, changes in the evolution with pressure of the full width half maximum (FWHM) of the (010) reflection takes place around 7.5 GPa, supporting that the phase-transition onset occurs near this pressure.

Additional information on the structural high-pressure behavior can be extracted from the study of the three triclinic angles of $\mathrm{CuWO}_{4}$. As shown in Fig. 4, a clear symmetrization is suffered by $\mathrm{CuWO}_{4}$ up to the onset of the phase transition with all the angles getting closer to $90^{\circ}$. Particularly interesting is the behavior of $\alpha$ and $\beta$ angles. Both decrease upon compression taking the same value when the phase-transition onset is detected. All these facts could be related with a symmetrization of the $\mathrm{CuO}_{6}$ octahedra associated to a reduction in the JT distortion. This phenomenon can be quantified through the pressure effects on the JT distortion parameter, defined as $\sigma_{\mathrm{JT}}=\sqrt{\frac{1}{6} \sum_{i=1}^{6}\left(R_{\mathrm{Cu}-\mathrm{O}}-\left\langle R_{\mathrm{Cu}-\mathrm{O}}\right\rangle\right)^{2}}$, where $R_{\mathrm{Cu}-\mathrm{O}}$ are the six $\mathrm{Cu}-\mathrm{O}$ distances of the $\mathrm{CuO}_{6}$ octahedra and $\left\langle R_{\mathrm{Cu}-\mathrm{O}}\right\rangle$ is the average $\mathrm{Cu}-\mathrm{O}$ distance. From our results, it can be deduced that $\sigma_{\mathrm{JT}}$ decreases from $0.201 \AA$ at ambient pressure to $0.160 \AA$ at $10 \mathrm{GPa}$, approaching the value of $\sigma_{\mathrm{JT}}$ in monoclinic wolframite-type $\mathrm{CdWO}_{4}(0.095 \AA)$ and $\mathrm{MnWO}_{4}$ $(0.088 \AA)$. This symmetrization of the $\mathrm{CuO}_{6}$ octahedra together with the fact that the triclinic structure of $\mathrm{CuWO}_{4}$ is a symmetry-reduced version of wolframite, suggest that the high-pressure phase (phase II) might have a monoclinic wolframite-type structure. This fact is consistent with present and previous experiments ${ }^{11}$ which found that the Raman spectra measured in phase II resemble very much those measured for wolframite $\mathrm{ZnWO}_{4}$ and $\mathrm{CdWO}_{4} \cdot{ }^{33,34}$

This hypothesis was used to analyze the diffraction data of phase II. We found that the diffraction patterns of phase II cannot be properly indexed considering only a wolframitetype phase $(P 2 / c, Z=2)$. However, we have been able to index the diffraction patterns assuming the coexistence of phases I and II (see Fig. 3). The unit-cell parameters obtained for both structures at $16 \mathrm{GPa}$ are given in Table I. Our results indicate that a volume change of about $1 \%$ occurs at the triclinic-monoclinic transformation. In view of this evidence, the proposed monoclinic structure appears as the most probable for the HP coexisting phase. This conclusion is also supported by $a b$ initio calculations as we will describe in Sec. IV B. The coexistence of both structures is compatible with the domain formation we detected by visual observation in single crystals. Macroscopic fringes are systematically observed at pressures close to the phase transition as can be seen in Fig. 6. Since the peak profile in x-ray diffraction experiments remained sharp throughout the coexistence range of phases I and II, pressure inhomogeneities or uniaxial stresses can be excluded as the origin of this phase coexistence. Instead, it could reflect a first-order character of the transition. This is further supported by the observed volume discontinuity at the phase transition. We would like to add here that the proposed structural sequence agrees with the systematic proposed for orthotungstates based upon crys-

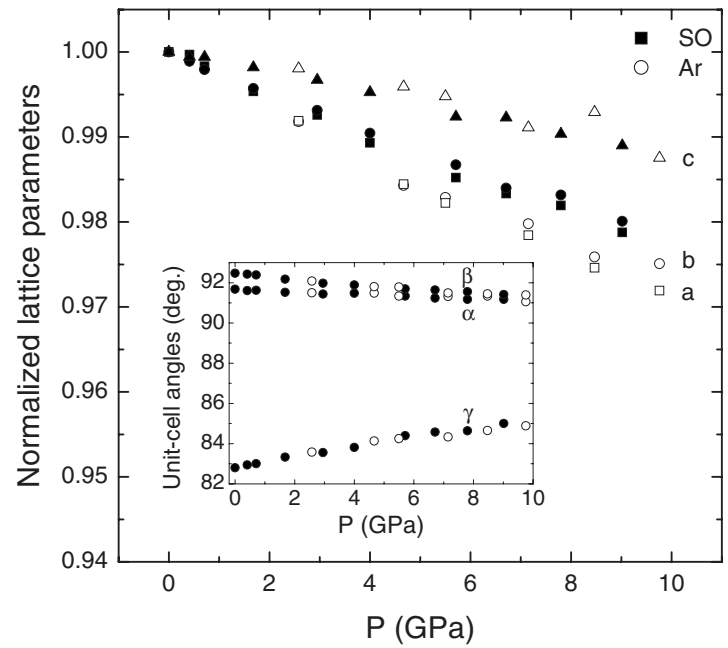

FIG. 4. Evolution of the normalized lattice parameters with pressure for both experiments: SO (solid symbols) and Ar (empty symbols). The unit-cell parameters are shown as (a) squares, (b) circles, and (c) triangles. The inset shows the pressure dependence of the triclinic angles. 


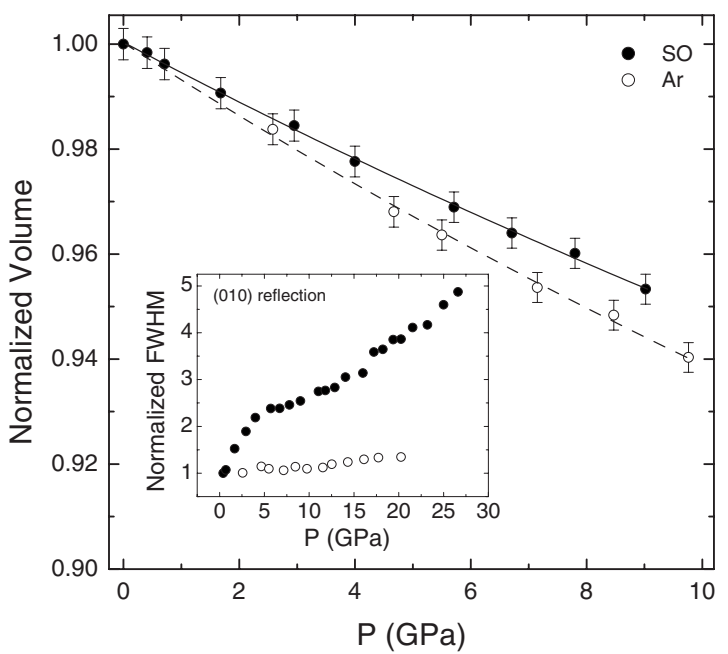

FIG. 5. Dependence of the normalized unit-cell volume with pressure for both experiments: SO (solid symbols) and Ar (empty symbols). Solid and dashed lines correspond to the EOS obtained from the $\mathrm{SO}$ and Ar data, respectively. The inset shows the variation in the normalized FWHM of the (100) Bragg peak of phase I with pressure.

tallochemical arguments. ${ }^{26}$ The phase transition is also consistent with the fact that in solid solutions of $\mathrm{CuWO}_{4}$ and $\mathrm{ZnWO}_{4}\left(\mathrm{NiWO}_{4}\right)$ an increase in the $\mathrm{Zn}(\mathrm{Ni})$ concentration induces a volume reduction and the transition from $\mathrm{cu}-$ proscheelite to wolframite at around $\mathrm{Zn}_{0.78} \mathrm{Cu}_{0.22} \mathrm{WO}_{4}$ $\left(\mathrm{Ni}_{0.6} \mathrm{Cu}_{0.4} \mathrm{WO}_{4}\right)^{6,35}$

Finally, we have to note that no information about the structural symmetry of phase III, observed in the experiment performed with SO, could be obtained from our ADXRD experiments. The broadening of diffraction peaks beyond 16 GPa together with the coexistence of phases precludes any reliable structural identification of phase III.

\section{B. Raman spectroscopy}

In order to complement the x-ray diffraction study of $\mathrm{CuWO}_{4}$, we explored its vibrational properties by means of Raman spectroscopy. Should the proposed phase coexistence
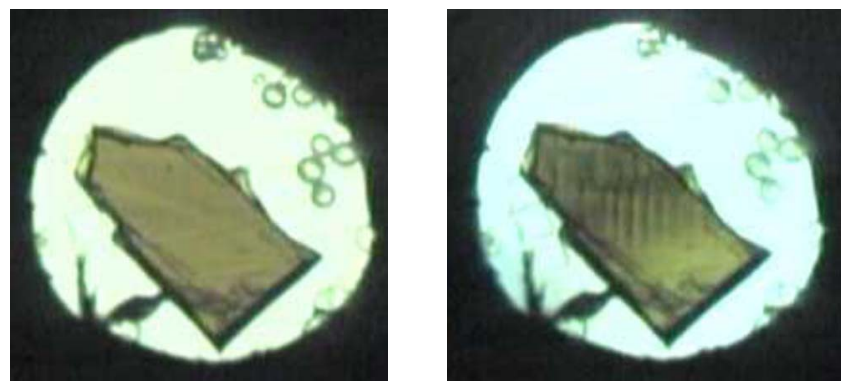

FIG. 6. (Color online) Images of a $\mathrm{CuWO}_{4}$ single crystal pressurized at 8.5 and $10.3 \mathrm{GPa}$ in a DAC using silicone oil as pressuretransmitting medium. At $8.5 \mathrm{GPa}$, the uniform brownish color indicates that only one phase is present. At $10.3 \mathrm{GPa}$, the color domains are due to the subtle phase transition and reveals two-phase coexistence.

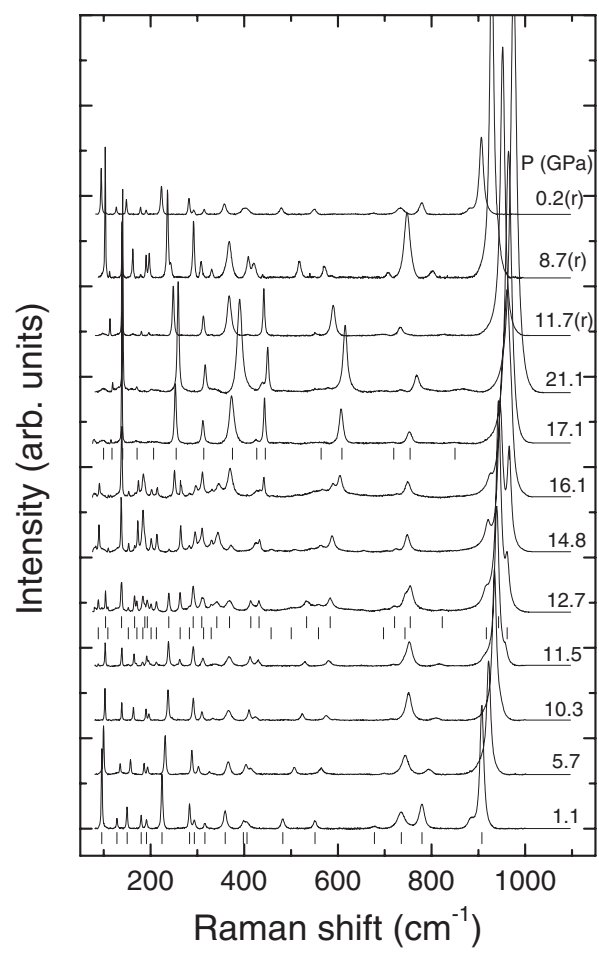

FIG. 7. Raman spectra of $\mathrm{CuWO}_{4}$ recorded at selected pressures. The ticks indicate the experimental modes assignation. At 12.7 $\mathrm{GPa}$, the upper ones are those assigned to the triclinic structure while the lower ones are those identified as wolframite $P 2 / c$ modes. Spectra collected with the LabRam setup.

be correct, we should observe up to 36 Raman modes, $18 \mathrm{~A}_{\mathrm{g}}$ corresponding to the triclinic phase plus $18\left(8 \mathrm{~A}_{\mathrm{g}}+10 \mathrm{~B}_{\mathrm{g}}\right)^{2}$ of wolframite. Figure 7 shows selected Raman spectra of $\mathrm{CuWO}_{4}$ up to $21 \mathrm{GPa}$. Tables II and III summarize the Raman modes of different phases at selected pressures. The Raman spectra up to $10.5 \mathrm{GPa}$ correspond to the lowpressure triclinic phase with its 18 Raman-active modes. ${ }^{11}$ Above this pressure, extra peaks appear whereas those of the low-pressure phase are still present. At $12.5 \mathrm{GPa}$, the number of peaks is already 36 as it was expected. This evidences the onset of a phase transition. Out of the 36 peaks, 18 can be assigned to the triclinic phase and the emerging 18 peaks are consistent with the HP monoclinic wolframite phase detected in x-ray diffraction experiments. These modes that appear after the phase transition resemble very much the Raman spectra of wolframite $\mathrm{CdWO}_{4}$ and $\mathrm{ZnWO}_{4} \cdot{ }^{33,34}$ Latticedynamics calculations confirm the assignment of the additional 18 modes detected for phase II to a monoclinic wolframite-type structure. Mode assignment was done based upon an analogy with ambient pressure wolframite tungstates and the fact that similar lattice-dynamics calculations successfully assign modes in them. ${ }^{33,34}$ However, the mode assignment should be fully confirmed by means of polarized Raman experiments. All these facts together with the x-ray diffraction analysis support the identification of the posttriclinic phase as a wolframite structure. The experimental frequencies of the Raman-active modes that correspond to phases I and II are indicated with ticks in Fig. 7 at different pressures. Note that additional changes leading to the appear- 
TABLE II. Raman modes, pressure coefficients, and Grüneisen parameters for phase I.

\begin{tabular}{|c|c|c|c|c|c|c|}
\hline \multicolumn{7}{|c|}{$P \overline{1}$} \\
\hline \multirow[b]{2}{*}{ Mode (sym) } & \multirow{2}{*}{$\begin{array}{c}\text { Literature } \\
\omega\left(1 \mathrm{bar}^{-1}\right) \\
\left(\mathrm{cm}^{-1}\right)\end{array}$} & \multicolumn{2}{|c|}{$A b$ initio } & \multicolumn{3}{|c|}{ Present experiment } \\
\hline & & $\begin{array}{c}\omega(1 \text { bar }) \\
\left(\mathrm{cm}^{-1}\right)\end{array}$ & $\begin{array}{c}d \omega / d P \\
\left(\mathrm{~cm}^{-1} / \mathrm{GPa}\right)\end{array}$ & $\begin{array}{c}\omega(1 \mathrm{bar}) \\
\left(\mathrm{cm}^{-1}\right)\end{array}$ & $\begin{array}{c}d \omega / d P \\
\left(\mathrm{~cm}^{-1} / \mathrm{GPa}\right)\end{array}$ & $\gamma$ \\
\hline $\mathrm{A}_{\mathrm{g}}$ & & 88.1 & 1.34 & 95.3 & 1.15 & 2.06 \\
\hline $\mathrm{A}_{\mathrm{g}}$ & & 115.6 & 1.84 & 127.6 & 1.65 & 2.21 \\
\hline$A_{g}$ & & 137.7 & 0.93 & 149.1 & 1.97 & 2.26 \\
\hline$A_{g}$ & 180 & 164.4 & 1.75 & 179.2 & 1.64 & 1.56 \\
\hline$A_{g}$ & 192 & 178.0 & 1.19 & 191.0 & 0.94 & 0.84 \\
\hline$A_{g}$ & 224 & 209.2 & 1.99 & 223.8 & 1.88 & 1.44 \\
\hline$A_{g}$ & 283 & 263.5 & 1.35 & 282.6 & 1.35 & 0.82 \\
\hline$A_{g}$ & 293 & 276.2 & 2.03 & 292.6 & 2.28 & 1.33 \\
\hline$A_{g}$ & 315 & 294.3 & 2.38 & 316.2 & 2.23 & 1.21 \\
\hline$A_{g}$ & 358 & 341.0 & 3.71 & 358.2 & 1.98 & 0.95 \\
\hline$A_{g}$ & 398 & 374.9 & 1.45 & 397.5 & 1.72 & 0.74 \\
\hline$A_{g}$ & 405 & 391.9 & 1.79 & 403.4 & 2.68 & 1.14 \\
\hline $\mathrm{A}_{\mathrm{g}}$ & 479 & 454.1 & 3.44 & 479.9 & 5.53 & 1.97 \\
\hline$A_{g}$ & 550 & 525.2 & 2.38 & 549.8 & 3.19 & 0.99 \\
\hline$A_{g}$ & 676 & 633.6 & 3.31 & 676.7 & 4.78 & 1.21 \\
\hline$A_{g}$ & 733 & 695.8 & 2.78 & 733.1 & 2.27 & 0.53 \\
\hline $\mathrm{A}_{\mathrm{g}}$ & 779 & 763.2 & 2.42 & 778.9 & 3.93 & 0.86 \\
\hline $\mathrm{A}_{\mathrm{g}}$ & 906 & 854.4 & 1.58 & 905.9 & 3.54 & 0.67 \\
\hline
\end{tabular}

ance of 16 different Raman modes occur in the Raman spectra at $17.1 \mathrm{GPa}$ confirming the occurrence of a second phase transition. Figure 8 shows a plot of the observed Raman peaks positions as a function of the pressure. A summary of the frequencies $(\omega)$ and pressure coefficients of the different modes for the three phases is given in Tables II and III. Table II also reports the Grüneisen parameters $\left(\gamma=B_{0} / \omega \cdot d \omega / d P\right)$ for the triclinic phase calculated using the bulk modulus obtained from ADXRD measurements using SO were used $\left[B_{0}=171(6) \mathrm{GPa}\right]$.

We would like to comment here that the coexistence of two phases which has been observed by means of both spectroscopic and structural techniques, could very well explain the previous hypothesis presented in Ref. 11 where a coexistence of $\mathrm{Cu}$ atoms in two atomic positions is argued. In that work, it is established that both positions would keep the $\mathrm{Cu}$ atoms octahedrally coordinated but while the large JahnTeller distortion would remain for one of the positions $(P \overline{1}$ structure) for the other one the $\mathrm{Cu}$ atom could be occupying a nearly $O_{\mathrm{h}}$ position (wolframite $P 2 / \mathrm{c}$ ) giving rise to a very well-defined $e_{\mathrm{g}} \rightarrow t_{2 \mathrm{~g}}$ optical absorption at the phase transition.

Regarding the second phase transition occurring above 17 $\mathrm{GPa}$, we have identified up to 16 modes in phase III (see Table III and Fig. 7). On pressure release phase III persists down to $8 \mathrm{GPa}$ where the Raman spectrum of the triclinic phase is recovered. The large hysteresis indicates that the second transition is strongly first order and points out the irreversibility of the phase III $\rightarrow$ phase II transition. Although we were unable to identify phase III from x-ray dif- fraction experiments, our Raman results could provide some important information regarding the building block and the coordination of the W-O and $\mathrm{Cu}-\mathrm{O}$ polyhedra. First, the Raman spectra of phase III do not show any resemblance to those of the post-wolframite phases in $\mathrm{CdWO}_{4}$ and $\mathrm{ZnWO}_{4} \cdot{ }^{33,34} \mathrm{In}$ particular, the most intense highest-frequency mode, which corresponds to the totally symmetric W-O stretching vibration, does not drop in frequency as typically occur at the HP phase transition in wolframites. This strongly suggests that no significant change is expected in the W-O coordination in the phase II-phase III transition, what is a worth information to identify the crystal structure of phase III.

\section{Calculations}

To help in the interpretation of our experimental results, $a b$ initio total-energy and lattice-dynamics calculations were performed for $\mathrm{CuWO}_{4}$. Along with the triclinic structure $(P \overline{1})$, a monoclinic wolframite-type structure $(P 2 / c)$ was considered. According to the calculations, the triclinic phase is the most stable structure from ambient pressure up to 9.3 GPa. Beyond this pressure a wolframite-type structure becomes energetically more favorable which is in good agreement with the phase transition detected in the experiments. For the triclinic structure at ambient pressure, the calculations gave $a=4.8352 \AA, b=6.0538 \AA, c=4.9429 \AA, \alpha$ $=93.26^{\circ}, \beta=94.25^{\circ}$, and $\gamma=80.11^{\circ}$. The obtained atomic positions are summarized in Table IV. The calculated unit-cell parameters are slightly larger than the experimental values. 
TABLE III. Raman modes and pressure coefficients for phases II and III.

\begin{tabular}{|c|c|c|c|c|c|c|}
\hline \multicolumn{5}{|c|}{$P 2 / c$} & \multirow{2}{*}{\multicolumn{2}{|c|}{$\begin{array}{c}\text { Phase III } \\
\text { Present experiment }\end{array}$}} \\
\hline & \multicolumn{2}{|c|}{ Ab initio } & \multicolumn{2}{|c|}{ Present experiment } & & \\
\hline Mode (sym) & $\begin{array}{c}\omega(12.3 \mathrm{GPa}) \\
\left(\mathrm{cm}^{-1}\right)\end{array}$ & $\begin{array}{c}d \omega / d P \\
\left(\mathrm{~cm}^{-1} / \mathrm{GPa}\right)\end{array}$ & $\begin{array}{c}\omega(12.7 \mathrm{GPa}) \\
\left(\mathrm{cm}^{-1}\right)\end{array}$ & $\begin{array}{c}d \omega / d P \\
\left(\mathrm{~cm}^{-1} / \mathrm{GPa}\right)\end{array}$ & $\begin{array}{c}\omega(17.1 \mathrm{GPa}) \\
\left(\mathrm{cm}^{-1}\right)\end{array}$ & $\begin{array}{c}d \omega / d P \\
\left(\mathrm{~cm}^{-1} / \mathrm{GPa}\right)\end{array}$ \\
\hline $\mathrm{B}_{\mathrm{g}}$ & 96.6 & 0.90 & 90.3 & 0.75 & 99.7 & 0.18 \\
\hline $\mathrm{A}_{\mathrm{g}}$ & 128.9 & -0.09 & 110.8 & 0.32 & 117.5 & 0.45 \\
\hline $\mathrm{B}_{\mathrm{g}}$ & 156.9 & 0.87 & 154.3 & 0.19 & 140.6 & 0.36 \\
\hline $\mathrm{B}_{\mathrm{g}}$ & 178.2 & 0.45 & 172.7 & 1.01 & 171.2 & -0.16 \\
\hline $\mathrm{B}_{\mathrm{g}}$ & 190.2 & 0.43 & 185.6 & 0.39 & 206.6 & 0.13 \\
\hline$A_{g}$ & 191.9 & 2.50 & 203.1 & 0.38 & 254.8 & 1.23 \\
\hline $\mathrm{A}_{\mathrm{g}}$ & 274.9 & 1.34 & 214.5 & 0.44 & 313.7 & 0.71 \\
\hline $\mathrm{B}_{\mathrm{g}}$ & 285.2 & 2.45 & 265.1 & 0.67 & 375.0 & 3.91 \\
\hline $\mathrm{A}_{\mathrm{g}}$ & 312.2 & 1.48 & 284.8 & 0.09 & 426.9 & 2.93 \\
\hline $\mathrm{B}_{\mathrm{g}}$ & 315.9 & 1.58 & 315.3 & 0.29 & 445.2 & 1.35 \\
\hline $\mathrm{B}_{\mathrm{g}}$ & 367.3 & 3.57 & 331.4 & 0.31 & 564.3 & 3.21 \\
\hline $\mathrm{A}_{\mathrm{g}}$ & 390.5 & 1.33 & 459.3 & 1.43 & 608.8 & 1.70 \\
\hline $\mathrm{B}_{\mathrm{g}}$ & 505.4 & 3.30 & 502.4 & 3.57 & 719.4 & 3.83 \\
\hline $\mathrm{A}_{\mathrm{g}}$ & 547.8 & 2.97 & 560.6 & 2.57 & 754.2 & 3.47 \\
\hline $\mathrm{B}_{\mathrm{g}}$ & 645.2 & 3.74 & 699.4 & 2.78 & 850.1 & 4.74 \\
\hline$A_{g}$ & 686.0 & 2.99 & 745.2 & 1.77 & 966.2 & 2.33 \\
\hline $\mathrm{B}_{\mathrm{g}}$ & 749.1 & 4.04 & 918.7 & 2.12 & & \\
\hline$A_{g}$ & 847.3 & 3.09 & 963.4 & 2.53 & & \\
\hline
\end{tabular}

This overestimation is within the typical reported systematic errors in DFT-GGA calculations. The calculated EOS of phase $\mathrm{I}$ is given by the following parameters: $B_{0}=77 \mathrm{GPa}$, $B_{0}^{\prime}=4$, and $V_{0}=142 \AA^{3}$. The obtained bulk modulus is $40 \%$ smaller than our experimental value. A possible reason for it

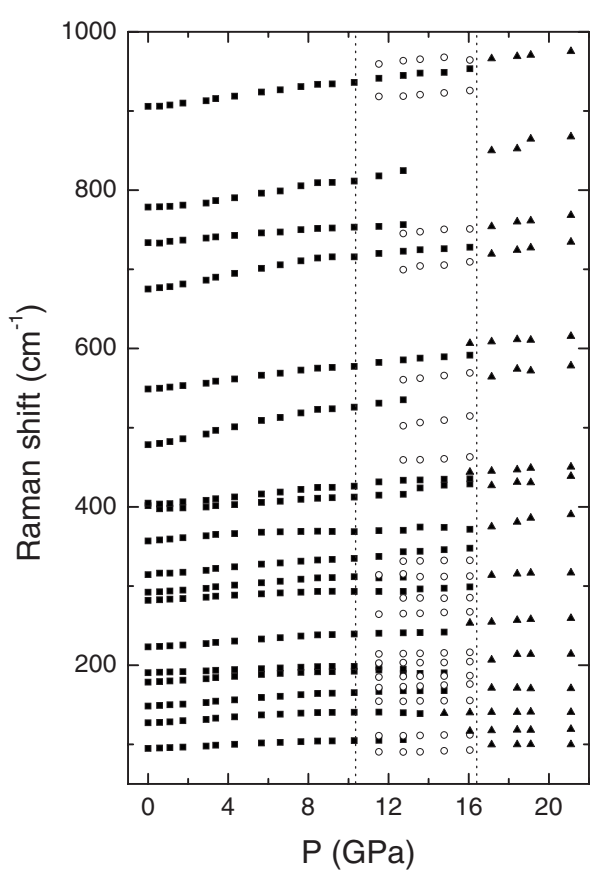

FIG. 8. Pressure dependence of the Raman-mode frequencies for different phases of $\mathrm{CuWO}_{4}$. is the known fact that GGA typically tends to underestimate the bulk modulus due to approximation used to calculate the exchange-correlation energy. ${ }^{36}$ In contrast, LDA calculation gives a bulk modulus of $134 \mathrm{GPa}$ closer to the experimental value; however its description of $\mathrm{CuWO}_{4}$ is very poor. Therefore, regarding high-pressure structural stability and phonons we will report the results obtained using GGA. The calculations also indicate that triclinic $\mathrm{CuWO}_{4}$ is magnetically ordered at $0 \mathrm{~K}$ having the equilibrium structure an $\mathrm{AF}$ state. $\mathrm{Cu}$ cations have a magnetic moment of $0.52 \mu_{\mathrm{B}}$ in good

TABLE IV. Calculated lattice parameters and internal coordinates for $\mathrm{CuWO}_{4}$.

\begin{tabular}{lcc}
\hline \hline & $P \overline{1}(0.3 \mathrm{GPa})$ & $P 2 / c(10.3 \mathrm{GPa})$ \\
\hline$a$ & 4.8352 & 4.5527 \\
$b$ & 6.0538 & 5.6479 \\
$c$ & 4.9429 & 4.9002 \\
$\alpha$ & 93.26 & 90.00 \\
$\beta$ & 94.25 & 90.06 \\
$\gamma$ & 80.11 & 90.00 \\
$\mathrm{Cu}$ & $2 \mathrm{i}(0.7523,0.5662,0.6569)$ & $2 \mathrm{f}(0.5,0.6641,0.25)$ \\
$\mathrm{W}$ & $2 \mathrm{i}(0.7899,0.5705,0.1228)$ & $2 \mathrm{e}(0,0.1802,0.25)$ \\
$\mathrm{O}_{1}$ & $2 \mathrm{i}(0.2663,0.9023,0.6204)$ & $4 \mathrm{~g}(0.2598,0.3817,0.4080)$ \\
$\mathrm{O}_{2}$ & $2 \mathrm{i}(0.2150,0.9538,0.0813)$ & $4 \mathrm{~g}(0.2251,0.8940,0.4302)$ \\
$\mathrm{O}_{3}$ & $2 \mathrm{i}(0.5095,0.7492,0.3471)$ & \\
$\mathrm{O}_{4}$ & $2 \mathrm{i}(0.9777,0.7434,0.8318)$ & \\
\hline \hline
\end{tabular}




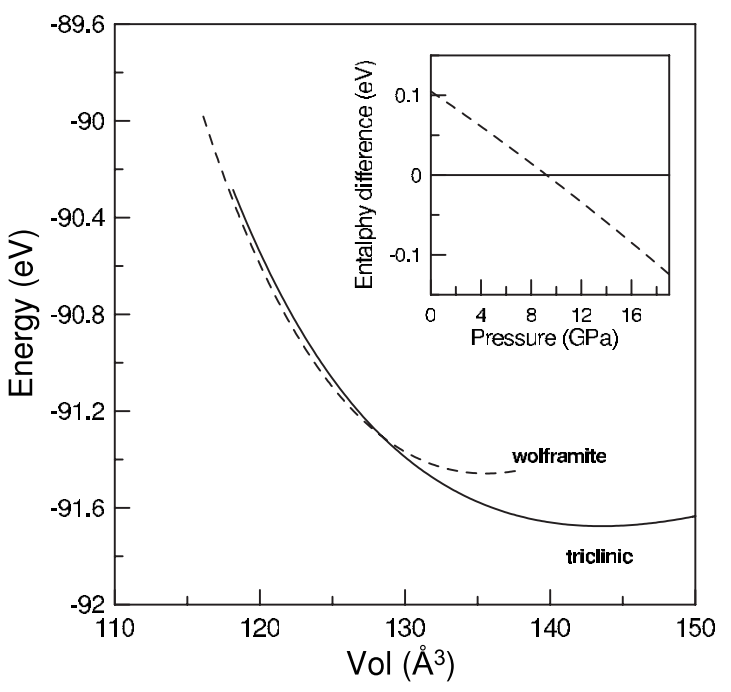

FIG. 9. Ab initio calculated energy vs volume curves for the triclinic and wolframite-type structures. The inset shows the enthalpy difference between both structures.

agreement with previous theoretical calculations ${ }^{37}$ and the $0.67 \mu_{\mathrm{B}}$ experimental value. ${ }^{38}$

In addition to the structural calculations, we have also performed lattice-dynamical calculations for phase I. Table II compares the calculated frequencies and pressure coefficients for the Raman modes with the experimental ones. The agreement between calculations and experiments for the lowpressure phase is reasonably good and similar to that obtained in $\mathrm{ZnWO}_{4}$ and $\mathrm{CdWO}_{4}{ }^{33,34}$ This fact supports the lattice-dynamics calculations performed for the highpressure phase of $\mathrm{CuWO}_{4}$. As it normally happens in these compounds the difference between experimental and calculated modes frequencies increases for the higher-energy modes. ${ }^{33,34}$

Considering now phase II, in our experiments we found evidence of a phase transition at $10 \mathrm{GPa}$ and the calculations found that at the same pressure, phase I becomes unstable against a wolframite-type phase (see Fig. 9). The structural parameters of the monoclinic phase are summarized in Table IV at $10.3 \mathrm{GPa}$. In fact if we compare the calculated lattice parameters $a=4.494 \AA, b=5.581 \AA, c=4.834 \AA$, and $\beta$ $=89.62^{\circ}$ at $16.8 \mathrm{GPa}$ with the experimental values showed in Table I at $16 \mathrm{GPa}$ we observe that the values are very similar. Moreover, according to the calculation in the phase II structure, the $\mathrm{CuO}_{6}$ octahedral distortion is very reduced showing a Jahn-Teller distortion of only $\sigma_{J T}^{\text {teo }}(10.3 \mathrm{GPa})=0.033 \AA$ when $\sigma_{\mathrm{JT}}=0$ means $O_{\mathrm{h}}$ symmetry. If we compare with the distortion showed by other wolframites and especially with the same arrangement in the triclinic phase, we could very well make the approximation of a nearly octahedral coordinated $\mathrm{CuO}_{6}$ with a completely quenched Jahn-Teller distortion in very good agreement with previous work hypothesis, ${ }^{11}$ as commented earlier in Sec. IV B. In contrast with the low-pressure phase, according with the calculations, phase II has a FM order at $0 \mathrm{~K}$. In this case $\mathrm{Cu}$ cations have a magnetic moment of $0.959 \mu_{\mathrm{B}}$. This fact implies that the triclinic-monoclinic transition goes beyond a mere structural transition involving also an AF-FM transition at low tem- peratures. Since strong spin-lattice interactions are determinant in the magnetic order of $\mathrm{CuWO}_{4}$ (Ref. 39) these interactions should be affected at the transition to allow the observed change in the magnetic order. Note that this change is analogous to findings in $\mathrm{CuMoO}_{4}$ whose monoclinic $\varepsilon-\mathrm{CuMoO}_{4}$ phase is FM ordered. ${ }^{40}$

From our calculations, we also determined the EOS for the HP phase, using a Birch-Murghnagam EOS we obtained the following parameters: $B_{0}=118 \mathrm{GPa}, B_{0}^{\prime}=6.7$, and $V_{0}$ $=135.1 \AA^{3}$. The calculated bulk modulus, for the wolframite structure of $\mathrm{CuWO}_{4}$, is similar to that of other wolframites and clearly suggests that the wolframite phase is much less compressible than the triclinic phase. Unfortunately, we cannot compare this result with the experiments (the phase coexistence observed beyond $10 \mathrm{GPa}$ does not allow to accurately determine the EOS for wolframite). Theoretical calculations suggest a volume reduction of $1.28 \%$ from phase I to phase II at the transition pressure.

In relation to the calculated Raman-mode frequencies and pressure coefficients for phase II, there are many mode frequencies that match very well the experimental values while for other modes the differences are bigger, especially for higher-frequency modes. The same thing happens for the pressure coefficients but in general the pressure behavior is well predicted. Finally, for phase III no calculations have been carried out since this third phase is only observed under nonhydrostatic conditions and it is therefore difficult to be predicted by $a b$ initio calculations.

\section{CONCLUDING REMARKS}

In summary, we have carried out two ADXRD experiments in the $\mathrm{CuWO}_{4}$ using two different pressuretransmitting media (SO and $\mathrm{Ar}$ ) up to $27 \mathrm{GPa}$ and Raman spectroscopy measurements up to $21 \mathrm{GPa}$ using MEW and $\mathrm{SO}$ as pressure-transmitting medium. Experimental measurements were complemented with $a b$ initio total-energy and lattice-dynamics calculations. We have obtained the compressibility of the material in both $\mathrm{SO}$ and Ar media showing a higher compressibility and anisotropy under quasihydrostatic conditions. In particular, an EOS is reported for $\mathrm{CuWO}_{4}$, being $B_{0}=139(6) \mathrm{GPa}$, a value similar to that of wolframite-structure tungstates. The detection of two phase transitions has been also reported at 10 and $16 \mathrm{GPa}$, with the second one only being detected under nonhydrostatic conditions. A possible structure for the high-pressure phase (phase II) is proposed and confirmed by the calculations, having this phase a monoclinic wolframite-type structure. Additionally, we found that pressure induces a reduction in the Jahn-Teller distortion in $\mathrm{CuWO}_{4}$, an enhancement of the symmetry of the low-pressure phase, as well as a possible Jahn-Teller quenching in the wolframite phase. Raman measurements, confirm the phase transitions observed by x-ray diffraction. We also determine the frequency and pressure dependence of all first-order modes of the low-pressure triclinic and highpressure phases. This phase transition has shown to be energetically favorable according to calculations, which additionally unravels the occurrence of an AF-FM phase transition together with the structural transformation. On top of that, 
TABLE V. Calculator IR modes for the monoclinic and triclinic phases of $\mathrm{CuWO}_{4}$.

\begin{tabular}{|c|c|c|c|c|c|}
\hline \multicolumn{3}{|c|}{$P \overline{1}$} & \multicolumn{3}{|c|}{$P 2 / c$} \\
\hline Mode(sym) & $\begin{array}{c}\omega(1 \mathrm{~atm}) \\
\left(\mathrm{cm}^{-1}\right)\end{array}$ & $\begin{array}{c}d \omega / d P \\
\left(\mathrm{~cm}^{-1} / \mathrm{GPa}\right)\end{array}$ & Mode(sym) & $\begin{array}{c}\omega(10.3 \mathrm{GPa}) \\
\left(\mathrm{cm}^{-1}\right)\end{array}$ & $\begin{array}{c}d \omega / d P \\
\left(\mathrm{~cm}^{-1} / \mathrm{GPa}\right)\end{array}$ \\
\hline $\mathrm{A}_{\mathrm{u}}$ & 0 & & $\mathrm{~B}_{\mathrm{u}}$ & 0 & \\
\hline $\mathrm{A}_{\mathrm{u}}$ & 0 & & $\mathrm{~B}_{\mathrm{u}}$ & 0 & \\
\hline $\mathrm{A}_{\mathrm{u}}$ & 0 & & $\mathrm{~A}_{\mathrm{u}}$ & 0 & \\
\hline $\mathrm{A}_{\mathrm{u}}$ & 101.4 & 5.09 & $\mathrm{~B}_{\mathrm{u}}$ & 138.3 & 1.40 \\
\hline $\mathrm{A}_{\mathrm{u}}$ & 157.3 & 0.72 & $\mathrm{~B}_{\mathrm{u}}$ & 200.8 & 0.87 \\
\hline $\mathrm{A}_{\mathrm{u}}$ & 214.2 & 0.23 & $\mathrm{~A}_{\mathrm{u}}$ & 204.8 & 2.70 \\
\hline $\mathrm{A}_{\mathrm{u}}$ & 239.0 & -1.64 & $\mathrm{~B}_{\mathrm{u}}$ & 228.5 & -1.73 \\
\hline $\mathrm{A}_{\mathrm{u}}$ & 266.1 & 1.37 & $\mathrm{~B}_{\mathrm{u}}$ & 258.7 & 1.72 \\
\hline $\mathrm{A}_{\mathrm{u}}$ & 281.0 & 0.71 & $\mathrm{~B}_{\mathrm{u}}$ & 299.9 & -0.23 \\
\hline $\mathrm{A}_{\mathrm{u}}$ & 320.5 & 1.27 & $\mathrm{~A}_{\mathrm{u}}$ & 300.6 & 3.33 \\
\hline $\mathrm{A}_{\mathrm{u}}$ & 332.3 & 1.98 & $\mathrm{~A}_{\mathrm{u}}$ & 332.1 & 1.13 \\
\hline $\mathrm{A}_{\mathrm{u}}$ & 383.8 & 4.21 & $\mathrm{~A}_{\mathrm{u}}$ & 433.3 & 3.01 \\
\hline $\mathrm{A}_{\mathrm{u}}$ & 438.9 & 2.67 & $\mathrm{~B}_{\mathrm{u}}$ & 466.2 & 4.98 \\
\hline $\mathrm{A}_{\mathrm{u}}$ & 474.8 & 4.03 & $A_{u}$ & 478.7 & 5.04 \\
\hline $\mathrm{A}_{\mathrm{u}}$ & 516.5 & 1.73 & $\mathrm{~B}_{\mathrm{u}}$ & 508.6 & 2.67 \\
\hline $\mathrm{A}_{\mathrm{u}}$ & 639.6 & 1.51 & $\mathrm{~A}_{\mathrm{u}}$ & 612.3 & 3.19 \\
\hline $\mathrm{A}_{\mathrm{u}}$ & 727.7 & 0.99 & $\mathrm{~B}_{\mathrm{u}}$ & 692.4 & 3.35 \\
\hline $\mathrm{A}_{\mathrm{u}}$ & 852.7 & 0.56 & $\mathrm{~A}_{\mathrm{u}}$ & 832.9 & 2.46 \\
\hline
\end{tabular}

lattice-dynamics calculations provided information about the Raman- and infrared-active modes as well as their HP behavior. The structure of the second HP phase (phase III) has not been identified so far but at least 16 modes have been identified for it. The Raman spectra of phase III suggest that W coordinated to six oxygen atoms as in phases I and II.

\section{ACKNOWLEDGMENTS}

Research financed by the Spanish MEC under Grants No. MAT2007-65990-C03-01, No. MAT2007-65990-C03-03, and No. CSD-2007-00045. Part of the experiments was conducted with the support of the Diamond Light Source at the I15 beamline (Proposal No. 683). Portions of this work were performed at HPCAT (16-IDB), Advanced Photon Source (APS), Argonne National Laboratory. HPCAT is supported by DOE-BES, DOE-NNSA, NSF, and the W.M. Keck Foun- dation. APS is supported by DOE-BES under Contract No. DE-AC02-06CH11357. The authors thank A. Kleppe and Y. Meng for technical support during the experiments. J.R.-F. and R.L.-P. thank the support from the MEC through the FPI and FPU programs. F.J.M. acknowledges financial support from Vicerrectorado de Investigación y Desarrollo de la UPV (Grant No. PAID-05-2009 through project UPV2010-0096). A.M. and P.R.-H. acknowledge the supercomputer time provided by the Red Española de Supercomputación (RES). J.R.-F., D.E., R.L.-P., A.S., J.G., F.R., F.J.M., S.R., P.R.-H., and A.M. are member of the MALTA Consolider Team.

\section{APPENDIX: INFRARED PHONONS}

For completion in Table $\mathrm{V}$ we present the IR-active modes for the triclinic and monoclinic phases as well as their pressure coefficients.

\footnotetext{
*Corresponding author; javier.ruiz-fuertes@uv.es

${ }^{1}$ M. Wiesmann, H. Ehrenberg, G. Miehe, T. Peun, H. Weitzel, and H. Fuess, J. Solid State Chem. 132, 88 (1997)

${ }^{2}$ D. Errandonea, Phys. Status Solidi B 242, R125 (2005).

${ }^{3}$ D. Errandonea, M. Somayazulu, and D. Häusermann, Phys. Status Solidi B 235, 162 (2003).

${ }^{4}$ J. Ruiz-Fuertes, S. López-Moreno, D. Errandonea, J. PellicerPorres, R. Lacomba-Perales, A. Segura, P. RodríguezHernández, A. Muñoz, A. H. Romero, and J. González, J. Appl.
}

Phys. 107, 083506 (2010).

${ }^{5}$ L. Kihlborg and E. Gebert, Acta Crystallogr., Sect. B: Struct. Crystallogr. Cryst. Chem. 26, 1020 (1970).

${ }^{6}$ P. F. Schofield and S. A. T. Redfern, J. Phys.: Condens. Matter 4, 375 (1992).

${ }^{7}$ S. A. T. Redfern, Phys. Rev. B 48, 5761 (1993).

${ }^{8}$ P. F. Schofield, J. M. Charnock, G. Gressey, and C. M. B. Henderson, Miner. Mag. 58, 185 (1994).

${ }^{9}$ P. F. Schofield, K. S. Knight, S. A. T. Redfern, and G. Gressey, 
Acta Crystallogr., Sect. B: Struct. Sci. 53, 102 (1997).

${ }^{10}$ J. Ruiz-Fuertes, D. Errandonea, A. Segura, F. J. Manjón, Zh. Zhu, and C. Y. Tu, High Press. Res. 28, 565 (2008).

${ }^{11}$ J. Ruiz-Fuertes, M. N. Sanz-Ortiz, J. González, F. Rodríguez, A. Segura, and D. Errandonea, J. Phys.: Conf. Ser. 215, 012048 (2010).

${ }^{12}$ D. Errandonea, R. Boehler, S. Japel, M. Mezouar, and L. R. Benedetti, Phys. Rev. B 73, 092106 (2006).

${ }^{13}$ A. Le Bail, Powder Diffr. 20, 316 (2005).

${ }^{14} \mathrm{G}$. Kresse, computer code VASP. See http://cms.mpi.univie.ac.at/ vasp

${ }^{15}$ G. Kresse and J. Hafner, Phys. Rev. B 47, 558 (1993).

${ }^{16}$ G. Kresse and J. Hafner, Phys. Rev. B 49, 14251 (1994).

${ }^{17}$ G. Kresse and J. Furthmüller, Comput. Mater. Sci. 6, 15 (1996).

${ }^{18}$ G. Kresse and J. Furthmüller, Phys. Rev. B 54, 11169 (1996).

${ }^{19}$ J. P. Perdew, K. Burke, and M. Ernzerhof, Phys. Rev. Lett. 77, 3865 (1996).

${ }^{20}$ P. E. Blöchl, Phys. Rev. B 50, 17953 (1994); G. Kresse and D. Joubert, ibid. 59, 1758 (1999).

${ }^{21}$ H. J. Monkhorst and J. D. Pack, Phys. Rev. B 13, 5188 (1976).

${ }^{22}$ F. J. Manjón, D. Errandonea, N. Garro, J. Pellicer-Porres, P. Rodríguez-Hernández, S. Radescu, J. López-Solano, A. Mujica, and A. Muñoz, Phys. Rev. B 74, 144111 (2006).

${ }^{23}$ K. Parlinski, computer code PHONON. See http://wolf.ifj.edu.pl/ phonon

${ }^{24}$ J. Macavei and H. Schulz, Z. Kristallogr. 207, 193 (1993).

${ }^{25}$ F. Birch, J. Geophys. Res. 83, 1257 (1978).

${ }^{26}$ D. Errandonea and F. J. Manjón, Prog. Mater. Sci. 53, 711 (2008).

${ }^{27}$ S. Klotz, J. C. Chervin, P. Munsch, and G. Le Marchand, J. Phys.
D 42, 075413 (2009).

${ }^{28}$ D. Errandonea, Y. Meng, M. Somayazulu, and D. Häusermann, Physica B 355, 116 (2005).

${ }^{29}$ N. Tateiwa and Y. Haga, Rev. Sci. Instrum. 80, 123901 (2009).

${ }^{30}$ M. Marqués, M. Flórez, J. M. Recio, L. Gerward, and J. Staun Olsen, Phys. Rev. B 74, 014104 (2006).

${ }^{31}$ H. Liu, Y. Ding, M. Somayazulu, J. Qian, J. Shu, D. Häusermann, and H.-K. Mao, Phys. Rev. B 71, 212103 (2005).

${ }^{32}$ R. Lacomba-Perales, D. Martínez-García, D. Errandonea, Y. Le Godec, J. Philippe, and G. Morard, High Press. Res. 29, 76 (2009).

${ }^{33}$ D. Errandonea, F. J. Manjón, N. Garro, P. Rodríguez-Hernández, S. Radescu, A. Mujica, A. Muñoz, and C. Y. Tu, Phys. Rev. B 78, 054116 (2008).

${ }^{34}$ R. Lacomba-Perales, D. Errandonea, D. Martinez-Garcia, P. Rodríguez-Hernández, S. Radescu, A. Mujica, A. Muñoz, J.-C. Chervin, and A. Polian, Phys. Rev. B 79, 094105 (2009).

${ }^{35}$ R. K. Selvan and A. Gedanken, Nanotechnology 20, 105602 (2009)

${ }^{36}$ A. Mujica, A. Rubio, A. Muñoz, and R. J. Needs, Rev. Mod. Phys. 75, 863 (2003).

${ }^{37}$ B. Lake, D. A. Tennant, R. A. Cowley, J. D. Axe, and C. K. Chen, J. Phys.: Condens. Matter 8, 8613 (1996).

${ }^{38}$ J. B. Forsyth, C. Wilkinson, and A. I. Zvyagin, J. Phys.: Condens. Matter 3, 8433 (1991).

${ }^{39}$ H. J. Koo and M. Whanegbo, Inorg. Chem. 40, 2161 (2001).

${ }^{40}$ J. Baek, A. S. Sefat, D. Mandrus, and P. S. Halasyamani, Chem. Mater. 20, 3785 (2008). 